TITLE:

\title{
Superconducting thin films of heavy-fermion compound CeColn5 prepared by molecular beam epitaxy
}

$\operatorname{AUTHOR}(S)$ :

Izaki, M; Shishido, H; Kato, T; Shibauchi, T; Matsuda, Y; Terashima, T

CITATION:

Izaki, M ... [et al]. Superconducting thin films of heavy-fermion compound CeColn5 prepared by molecular beam epitaxy. APPLIED PHYSICS LETTERS 2007, 91(12): 122507.

ISSUE DATE:

2007-09-17

URL:

http://hdl.handle.net/2433/49824

RIGHT:

Copyright 2007 American Institute of Physics. This article may be downloaded for personal use only. Any other use requires prior permission of the author and the American Institute of Physics. 


\title{
Superconducting thin films of heavy-fermion compound CeColn ${ }_{5}$ prepared by molecular beam epitaxy
}

\author{
M. Izaki, ${ }^{\text {a) }}$ H. Shishido, T. Kato, and T. Shibauchi \\ Department of Physics, Kyoto University, Sakyo-ku, Kyoto 606-8502, Japan \\ Y. Matsuda \\ Department of Physics, Kyoto University, Sakyo-ku, Kyoto 606-8502, Japan and Institute for Solid State \\ Physics, University of Tokyo, Kashiwa, Chiba 277-8581, Japan \\ T. Terashima \\ Research Center for Low Temperature and Materials Sciences, Kyoto University, Uji, Kyoto 611-0011, \\ Japan
}

(Received 17 July 2007; accepted 30 August 2007; published online 20 September 2007)

\begin{abstract}
Highly $c$-axis oriented thin films of Ce-based heavy-fermion superconductor $\mathrm{CeCoIn}_{5}$ were grown on $\mathrm{Cr} / \mathrm{MgO}$ substrates by molecular beam epitaxy. The films consist of micrograins which are strongly oriented in $c$ axis but are random in the film plane. Kondo temperature, superconducting transition temperature, and upper critical fields of the films are very close to those of single crystals. (C) 2007 American Institute of Physics. [DOI: 10.1063/1.2787969]
\end{abstract}

Superconductivity of heavy-fermion (HF) systems is a key subject in condensed matter physics because its variegated aspect concerns an interplay between magnetism and superconductivity. Particularly, Ce-based intermetallic compounds exhibit a rich variety of properties depending on the interaction between $4 f$ and conduction electrons. Electronic state in Ce-based compounds is dominated by the competition between the Rudermann-Kittel-Kasuya-Yoshida interaction and the Kondo effect. It has been found that several Ce-based compounds which are in the HF state often show superconductivity. ${ }^{1}$

Recently, a new class of HF compounds with the chemical formula $\mathrm{CeMIn}_{5}(M=\mathrm{Rh}$, Ir, or Co $)$ have been discovered. ${ }^{2}$ The electronic structure of these compounds is quasi-two-dimensional, as revealed, e.g., by de Haas-van Alphen measurements. ${ }^{3}$ In particular, $\mathrm{CeCoIn}_{5}$ is a superconductor with the highest transition temperature $\left(T_{c}=2.3 \mathrm{~K}\right)$ among Ce-based HF superconductors. The superconductivity in $\mathrm{Ce} M \mathrm{In}_{5}$ appears in the vicinity of the antiferromagnetic metallic state. The physical properties of $\mathrm{CeCoIn}_{5}$ have attracted considerable attention. In the normal state, striking deviation from the Fermi liquid behavior has been reported. ${ }^{4,5}$ In the superconducting state, the gap symmetry most likely belongs to the $d$-wave class. ${ }^{6-9}$ Therefore, it seems to be widely accepted that the magnetic fluctuation is responsible for the pairing interaction, in contrast to conventional superconductors. ${ }^{10}$ Furthermore, in magnetic fields, an exotic Fulde-Ferrell-Larkin-Ovchinnikov state with a spatial oscillation of the superconducting order parameter, has been reported. ${ }^{11-13}$

Thus far, HF materials have been studied mainly by using bulk crystals. A few exceptions include $\mathrm{UPd}_{2} \mathrm{Al}_{3}$ superconductor, which has been prepared as an epitaxial thin film, ${ }^{14,15}$ and nonsuperconducting $\mathrm{CeCu}_{6}$ thin films, which have been prepared in polycrystalline forms by a magnetron sputtering method. ${ }^{16}$ Thin films are important to fabricate various heterostructures including $\mathrm{S} / \mathrm{I} / \mathrm{S}, \mathrm{S} / \mathrm{N} / \mathrm{S}$, and $\pi$

\footnotetext{
${ }^{a)}$ Electronic mail: izaki@msk.kuicr.kyoto-u.ac.jp
}

junctions, ${ }^{17}$ and have various advantages to study the fundamental physical properties in the superconducting state of $\mathrm{CeCoIn}_{5}$.

In this letter, we report the preparation, structure, and superconducting properties of $\mathrm{CeCoIn}_{5}$ thin films. We have grown highly $c$-axis oriented thin films of $\mathrm{CeCoIn}_{5}$ by a molecular beam epitaxy (MBE) method. The resistivity behaviors of our thin films reproduced well the characteristic features of $\mathrm{CeCoIn}_{5}$ bulk single crystals.

$\mathrm{CeCoIn}_{5}$ thin films were grown by MBE method. The base pressure of the MBE apparatus was less than 1 $\times 10^{-7} \mathrm{~Pa}$ and the pressure during the growth was maintained below $10^{-6} \mathrm{~Pa}$. The elementary metal components $\mathrm{Ce}$ (99.9\%), Co (99.99\%), and In (99.9999\%) were evaporated from Knudsen cells ( $K$ cells). Especially, for Ce and Co, high temperature-type $K$ cells were used. The choice of crucible material is a crucial matter for rare-earth elements because of their high chemical reactivity. We used Ta as a crucible material for Ce. The evaporation rate of each element was monitored by an oscillating quartz crystal monitor located below the substrate. The rate of $\mathrm{Ce}, \mathrm{Co}$, and $\mathrm{In}$ were 1.5 $\times 10^{-2}, 1.5 \times 10^{-2}$, and $6.0 \times 10^{-2} \mathrm{~mol} / \mathrm{s}$, respectively.

$\mathrm{CeCoIn}_{5}$ crystallizes in tetragonal $\mathrm{HoCoGa}_{5}$-type structure in which two-dimensional $\mathrm{CeIn}_{3}$ and $\mathrm{CoIn}_{2}$ layers are alternately stacked along the [001] direction. Lattice constants along $a$ and $c$ axes are 0.462 and $0.756 \mathrm{~nm}$, respectively. Here, we examined $\mathrm{MgO}$ (100) whose lattice mismatch is as large as 10\%. Furthermore, 10-nm-thick chromium buffer layer grown on $\mathrm{MgO}$ (100) was used as a substrate. The smoothness of the substrate surface could be improved by the deposition of chromium thin film. Chromium grows epitaxially on $\mathrm{MgO}$ (100) with $[110]_{\mathrm{Cr}} \|[100]_{\mathrm{MgO}}$ and $(100)_{\mathrm{Cr}} \|(100)_{\mathrm{MgO}}$. The $\mathrm{MgO}$ substrate surface was etched chemically by $\mathrm{HNO}_{3}$ /ethanol $(0.05 \%)$ solution and was rinsed by acetone and hexane. The substrate was heated at $450{ }^{\circ} \mathrm{C}$ before the deposition and the films were deposited at $350{ }^{\circ} \mathrm{C}$.

The structure of the film was investigated by $\mathrm{x}$-ray diffraction method. The composition of the film was investigated by energy dispersive $\mathrm{x}$-ray (EDX) spectroscopy. The 


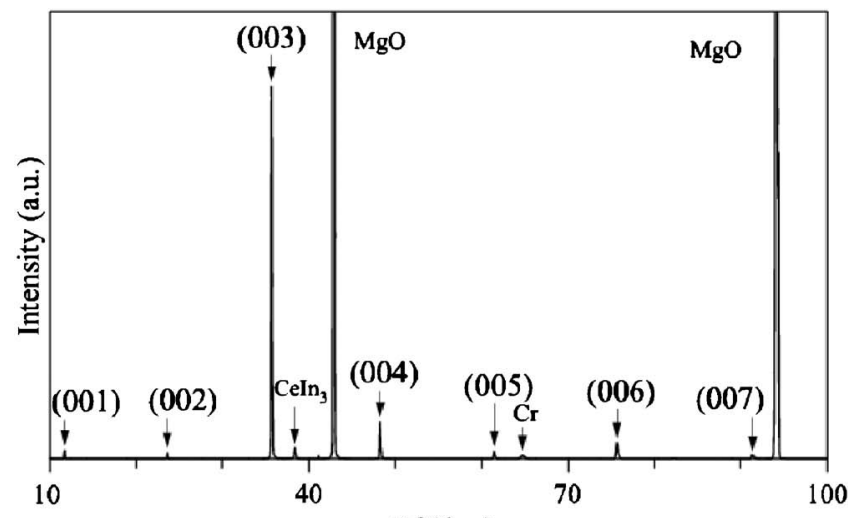

(a)

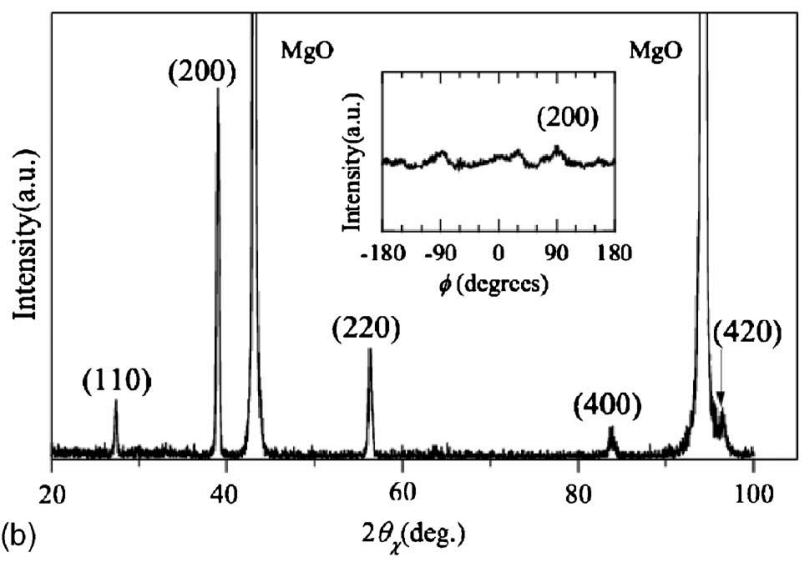

FIG. 1. (a) X-ray diffraction pattern for a 400-nm-thick $\mathrm{CeCoIn}_{5}$ film with a scattering vector normal to the film plane. (b) Grazing-incidence x-ray diffraction pattern for the same film as in (a). The inset shows $\phi$ scan of the (200) peak.

resistivity measurement was done by a conventional fourprobe method. 40-nm-thick Au pads were deposited on $\mathrm{CeCoIn}_{5}$ thin films as electric contacts. The current is applied along the film plane with the current density of $0.6 \mathrm{~A} / \mathrm{cm}^{2}$.

Figure 1 (a) shows the $\theta-2 \theta$ x-ray diffraction profile of the 400-nm-thick $\mathrm{CeCoIn}_{5}$ film grown on $\mathrm{Cr}(100) / \mathrm{MgO}(100)$ substrate with a scattering vector normal to the film plane. Appearance of only $(00 l)$ peaks of $\mathrm{CeCoIn}_{5}$ phase revealed that the film has a distinct $c$-axis oriented structure. The intensity ratio of the $(00 l)$ peaks well agrees with simulated one which assumes the $c$-axis orientation with lattice and atomic position parameters of bulk crystal. Small trace of $\mathrm{CeCoIn}_{5}$ phase is seen in the profile, indicating a slight misadjustment in composition control. From $(00 l)$ diffraction peaks, $c$-axis lattice constant is determined to be $0.753 \mathrm{~nm}$ which is in fairly good agreement with the bulk value within $0.2 \%$. Definite x-ray diffraction pattern was not detected for thin films grown directly on $\mathrm{MgO}$ (100) substrate, which indicates that the $\mathrm{Cr}$ buffer layer plays an important role for the nucleation of $\mathrm{CeCoIn}_{5}$ crystal.

Grazing-incidence x-ray diffraction profile for the film is shown in Fig. 1(b). Observed diffraction peaks (110), (200), (220), (400), and (440) originate from (00l) oriented grains. $a$-axis lattice constant is determined to be $0.462 \mathrm{~nm}$. $\phi$ scan of (200) peak is displayed in the inset of Fig. 1(b). The structureless profile of the diffraction indicates that the inplane axes of the grains are randomly oriented. This is most likely due to the large mismatch value, $13 \%$, between $\mathrm{Cr}$ and $\mathrm{CeCoIn}_{5}$. It is assumed that the crystal growth of $\mathrm{CeCoIn}_{5}$ Downloaded 05 Mar 2008 to 130.54 .110 .22 . Redistribution subject

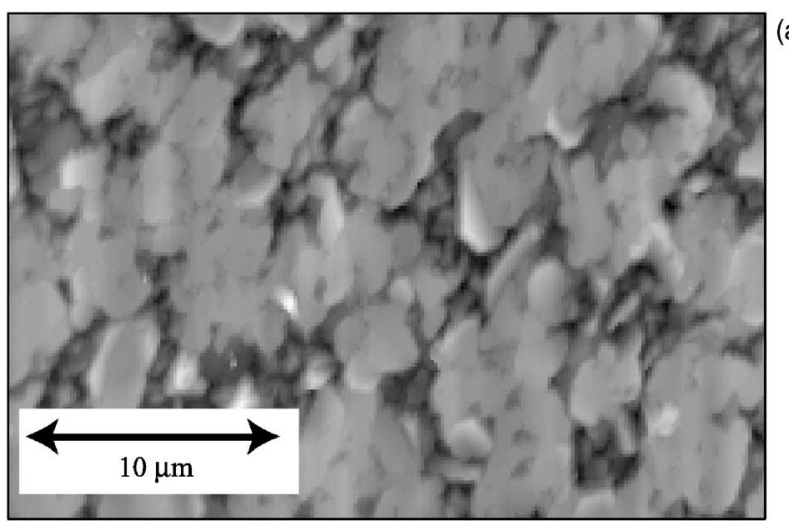

(a)

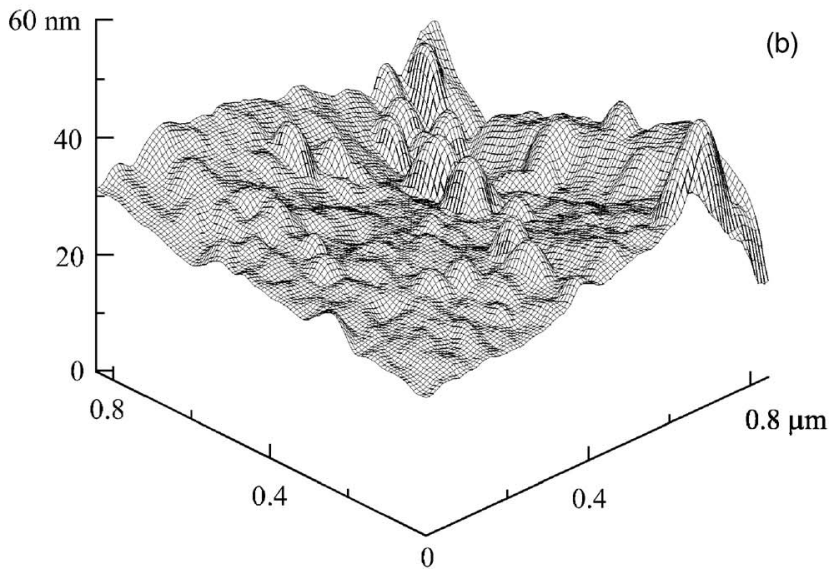

FIG. 2. (a) AFM image for the 400-nm-thick CeCoIn ${ }_{5}$ film. (b) 3D plot for the surface of a single grain.

occurs without any constraint from the substrate.

Atomic-force microscope (AFM) image of the $\mathrm{CeCoIn}_{5}$ film is displayed in Fig. 2(a). The film consists of micrometer-sized grains. The edge of the grains are presum-

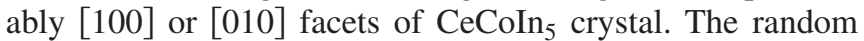
in-plane orientation of the grains is in agreement with the results of x-ray diffraction. Figure 2(b) shows the threedimensional (3D) imaging picture of a single grain. The roughness of the surface is within $4 \mathrm{~nm}$, while some protuberances are seen in the image.

By optimizing the evaporation rate, $\mathrm{Ce} / \mathrm{Co}$ ratio could be adjusted to nearly 1.0, whereas a control of In flux is rather difficult, which leads to a fluctuation of the ratio to other metals from 4.0 to 5.0. We also found from EDX that the intergranular regions have smaller compositions of $\mathrm{Co}$ and In relative to $\mathrm{Ce}$.

We have observed in situ reflection high-energy electron diffraction (RHEED) during the film growth. After initiating the growth, RHEED pattern immediately disappeared and never reappeared. This means that the growing surface of the film is too rough to reflect the electron beam with a small incidence angle. These results suggest that the development of more suitable substrate is indispensable to prepare epitaxial thin films.

Figure 3(a) shows the temperature dependence of resistivity $\rho(T)$ for the 400-nm-thick $\mathrm{CeCoIn}_{5}$ thin film. The order of magnitude and overall temperature dependence of $\rho(T)$ show fairly good agreement with bulk data. With decreasing temperature, the resistivity increases gradually at high temperatures due to the Kondo effect. At low temperatures, the resistivity decreases linearly after showing a broad peak at AIP license or copyright; see http://apl.aip.org/apl/copyright.jsp 

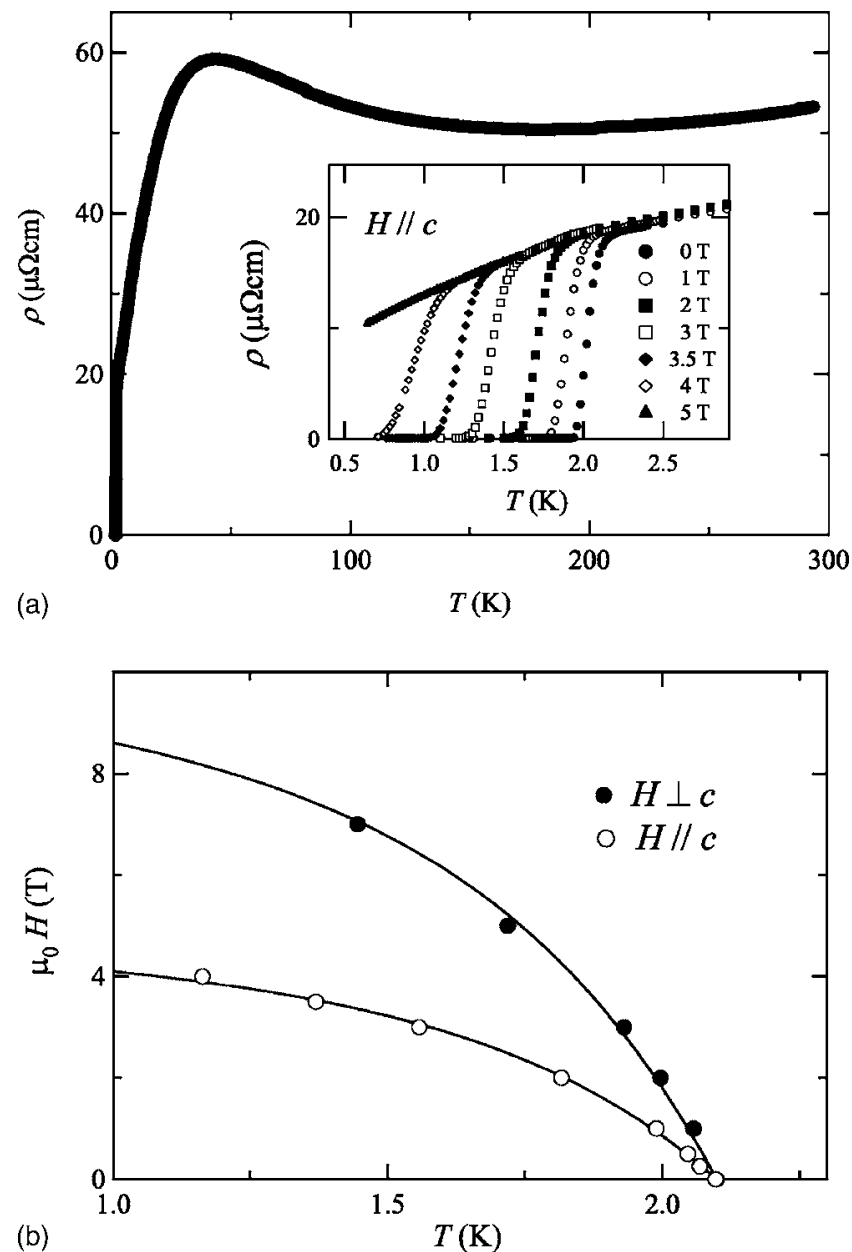

FIG. 3. (a) Temperature dependence of the resistivity for the 400-nm-thick $\mathrm{CeCoIn}_{5}$ film. The inset is the expanded view near the superconducting transition under magnetic fields applied along the $c$ axis. (b) Temperature dependence of upper critical fields for $H \| c$ (open circles) and for $H \perp c$ (solid circles). The solid lines are the guides for the eyes.

$\sim 30 \mathrm{~K}$. A superconducting transition occurs at $\sim 2.2 \mathrm{~K}$ with slightly broader transition width $\Delta T_{c}(10 \%-90 \%) \sim 0.2 \mathrm{~K}$ than that of high-quality bulk crystals (see inset). The resistive transition shifts to lower temperatures under magnetic fields, and the temperature dependence of upper critical field $H_{c 2}$ is extracted from the onset temperatures of the transition. As shown in Fig. 3(b), $H_{c 2}$ for two field orientations have clear anisotropy of $\sim 2.1$, which is also in good correspondence with the bulk results, ${ }^{11}$ confirming the highly $c$-axis orientated nature of the films. This also ensures the dominant intragrain conduction of our superconducting films, which is consistent with the fact that the grain size is much larger than the coherence length $\xi_{a b} \sim 5 \mathrm{~nm}$.

In summary, we have prepared highly $c$-axis oriented thin films of superconducting $\mathrm{CeCoIn}_{5}$ on $\mathrm{Cr}(100) / \mathrm{MgO}(100)$ substrate. Resistive behaviors of the film are in good agreement with those of bulk crystals. To investigate further the fundamental superconducting properties and fabricate various type junctions, high-quality epitaxial thin films with well oriented crystal axes in the film plane are strongly required, which deserves further studies.

This work was partly supported by Grants-in-Aid for Scientific Research from Japan Society for the Promotion of Science (JSPS). H.S. was supported by the Research Fellowship of JSPS for Young Scientists. After completion of our work, we became aware of recent MBE study of $\mathrm{CeCoIn}_{5}$ films on $\mathrm{Al}_{2} \mathrm{O}_{3}$ substrates. ${ }^{18}$

${ }^{1}$ P. Thalmeier and G. Zwicknagl, Handbook on the Physics and Chemistry of Rare Earths (North-Holland, Amsterdam, 2005), Vol. 34, Chap. 219, p. 135.

${ }^{2}$ C. Petrovic, P. G. Pagliuso, M. F. Hundley, R. Movshovich, J. L. Sarrao, J. D. Thompson, Z. Fisk, and P. Monthoux, J. Phys.: Condens. Matter 13, L337 (2001)

${ }^{3}$ H. Shishido, R. Settai, D. Aoki, S. Ikeda, H. Nakawaki, N. Nakamura, T. Iizuka, Y. Inada, K. Sugiyama, T. Takeuchi, K. Kindo, T. C. Kobayashi, Y. Haga, H. Harima, Y. Aoki, T. Namiki, H. Sato, and Y. Ōnuki, J. Phys. Soc. Jpn. 71, 162 (2002).

${ }^{4}$ Y. Nakajima, H. Shishido, H. Nakai, T. Shibauchi, K. Behnia, K. Izawa, M. Hedo, Y. Uwatoko, T. Matsumoto, R. Settai, Y. Ōnuki, H. Kontani, and Y. Matsuda, J. Phys. Soc. Jpn. 76, 024703 (2007).

${ }^{5}$ Y. Nakajima, K. Izawa, Y. Matsuda, S. Uji, T. Terashima, H. Shishido, R. Settai, Y. Ōnuki, and H. Kontani, J. Phys. Soc. Jpn. 73, 5 (2004).

${ }^{6}$ R. Movshovich, M. Jaime, J. D. Thompson, C. Petrovic, Z. Fisk, P. G. Pagliuso, and J. L. Sarrao, Phys. Rev. Lett. 86, 5152 (2001).

${ }^{7}$ K. Izawa, H. Yamaguchi, Y. Matsuda, H. Shishido, R. Settai, and Y. Ōnuki, Phys. Rev. Lett. 87, 057002 (2001).

${ }^{8}$ Y. Kohori, Y. Yamato, Y. Iwamoto, T. Kohara, E. D. Bauer, M. B. Maple, and J. L. Sarrao, Phys. Rev. B 64, 134526 (2001).

${ }^{9}$ R. J. Ormeno, A. Sibley, C. E. Gough, S. Sebastian, and I. R. Fisher, Phys. Rev. Lett. 88, 047005 (2001).

${ }^{10}$ Y. Matsuda, K. Izawa, and I. Vekhter, J. Phys.: Condens. Matter 18, R705 (2006).

${ }^{11}$ A. Bianchi, R. Movshovich, C. Capan, P. G. Pagliuso, and J. L. Sarrao, Phys. Rev. Lett. 91, 187004 (2003).

${ }^{12}$ K. Kakuyanagi, M. Saitoh, K. Kumagai, S. Takashima, M. Nohara, H. Takagi, and Y. Matsuda, Phys. Rev. Lett. 94, 047602 (2005).

${ }^{13}$ Y. Matsuda and H. Shimahara, J. Phys. Soc. Jpn. 76, 051005 (2007).

${ }^{14}$ M. Jourdan, M. Huth, and H. Adrian, Nature (London) 398, 47 (1999).

${ }^{15}$ M. Huth, A. Kaldowski, J. Hessert, Th. Steinborn, and H. Adrian, Solid State Commun. 87, 1133 (1993).

${ }^{16}$ D. Groten, G. J. C. van Baarle, J. Aarts, G. J. Nieuwenhuys, and J. A. Mydosh, Phys. Rev. B 64, 144425 (2001).

${ }^{17}$ C. C. Tsuei and J. R. Kirtley, Rev. Mod. Phys. 72, 969 (2000).

${ }^{18}$ O. K. Soroka, G. Blendin, and M. Huth, J. Phys.: Condens. Matter 19, 056006 (2007) 